$\underline{\text { Iranian Journal of Pathology | ISSN: 2345-3656 }}$

\title{
Prognostic Significance of Reduction in Ki67 Index After Neoadjuvant Chemotherapy in Patients With Breast Cancer in Kerman Between 2009 And 2014
}

\author{
Vahid Moazed ${ }^{1}$, Elham Jafari ${ }^{2 *}$, Behjat Kalantari Khandani ${ }^{1}$, Ali Nemati ${ }^{1}$, Alale Roozdar ${ }^{2}$, \\ Seyyed Amir Ben Razavi ${ }^{3}$ \\ 1. Hematology and Oncology Division, Dept. of Internal medicine, Afzalipour Kerman Medical Science University, Kerman, Iran \\ 2. Pathology and Stem Cells Research Center, Dept. of Pathology, Afzalipour Kerman Medical Science University, Kerman, Iran \\ 3. Dept. of Internal Medicine, Afzalipour Kerman Medical Science University, Kerman, Iran

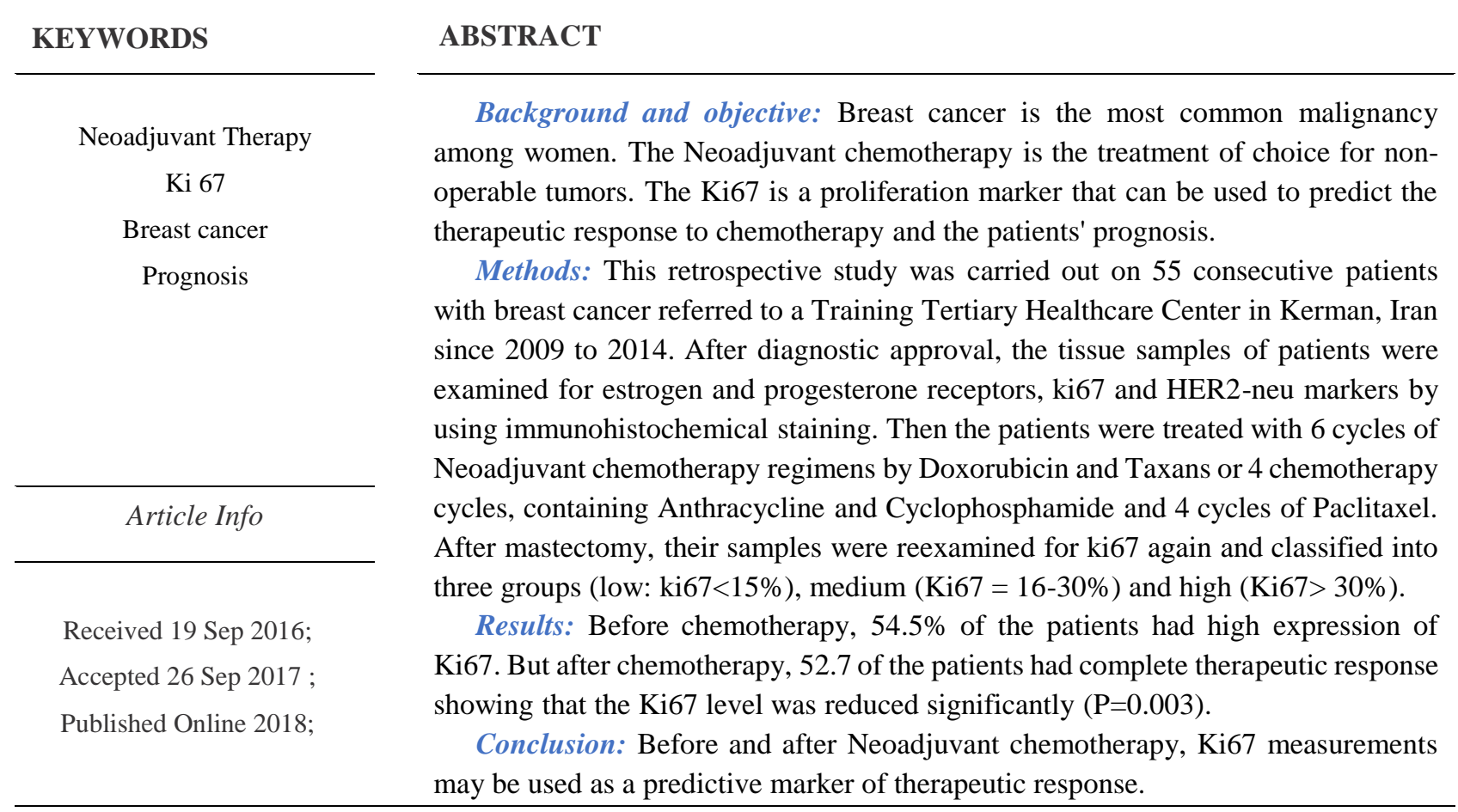

Corresponding information: Elham Jafari, Associate professor of pathology, Pathology and stem cells research center, Pathology Department, Afzalipour Kerman Medical Science university, Kerman, Iran, Email: ejfarda@ yahoo.com

Copyright ( 2017 , IRANIAN JOURNAL OF PATHOLOGY. This is an open-access article distributed under the terms of the Creative Commons Attribution-noncommercial 4.0 International License which permits copy and redistribute the material just in noncommercial usages, provided the original work is properly cited.

\section{Introduction}

Breast cancer is the most common cancer in women worldwide and also in Iran, it is the fifth leading cause of cancer-related deaths among Iranian women especially in the age group of 49 to 79 years $(1,2)$. Unfortunately, the age of breast cancer onset in Iranian women is several years earlier than those in other countries resulting in delayed diagnosis $(3,4)$. Neoadjuvant chemotherapy is a therapeutic option for inoperable tumors and also in patients who are candidate for breast conserving surgery (5). To choose the best therapeutic option, determination of prognostic factors is crucial. Ki67 is a proliferative marker which may be used to determine not only the chemotherapy regimen but also can predict the therapeutic response to Neoadjuvant therapy (6, $7)$.

High level of Ki67 is related to low survival rate in breast cancer patients (8) and low levels of this marker can predict better therapeutic response to Neoadjuvant therapy $(9,10)$. Neoadjuvant chemotherapy causes decline in Ki67 expression in breast cancer specimens (11). So Ki67 level is a prognostic factor to determine the patients' status after chemotherapy in short and long-term follow up $(12,13)$. Also Ki67 is a predictive factor for pathologic complete response in cancer patients with estrogen receptor-negative and HER2/neupositive status (6). Post-treatment Ki67 levels 
provide better prognostic information and high Ki67 levels after the treatment would reveal further treatment requirement after Neoadjuvant chemotherapy (14). Patients with higher level of Ki67 have higher risk of tumor recurrence and death $(15,16)$.

So far, there is no conducted study to investigate the prognostic value of reduction in Ki67 levels after chemotherapy in Iranian population. However this research can also play an important role to determine how long the treatment should be continued, this study was performed to assess the prognostic significance of decreased ki67index after Neoadjuvant chemotherapy in breast cancer patients in Kerman between the years of 2009 and 2014.

\section{Materials and methods}

This retrospective study was performed on 55 female patients with approved breast cancer relying on core needle biopsy specimen from breast masses. The tissue specimens underwent IHC staining (using DAKO standard method) for ER, PR, HER2/neu and Ki67 markers. Four molecular groups were as follow;

Luminal A: ER positive, Her2/neu negative, low Ki67 (lower than 14\%) and high PR levels. This subtype is solely cured with endocrine treatment. But chemotherapy can be applied to patients with several lymph nodes or large tumor bulk.

Luminal B: Her2/neu negative, It is associated with ER positive and either high $\mathrm{Ki} 67$ (higher than 14\%) or low PR.

Luminal B like: HER2/neu positive, ER positive that show high expression of HER2/neu and any levels of Ki67 or PR.

HER2/neu positive: over expression or high expression of HER2/neu, ER negative and PR

Triple negative: ER, PR and HER2/neu negative (17)

Patients were treated using 6 cycles of Neo adjuvant chemotherapy with Doxorubicin and Taxans, 4 cycles of chemotherapy including Anthracycline and Cyclophosphamide and 4 cycles of Paclitaxel. The patients then underwent the breast mastectomy for two weeks after finishing their chemotherapy regiments. Their tissue specimen after surgery were fixed in formalin and blocked in paraffin, they were also sectioned and then stained with IHC for Ki67 nuclear marker.

Patients who did not underwent mastectomy after chemotherapy or those who received chemotherapy regimen in center were excluded from the present study. Slides of ER, PR, HER2/neu markers and also Ki67 were inspected before and after chemotherapy and scored by a pathologist (Figures 1)

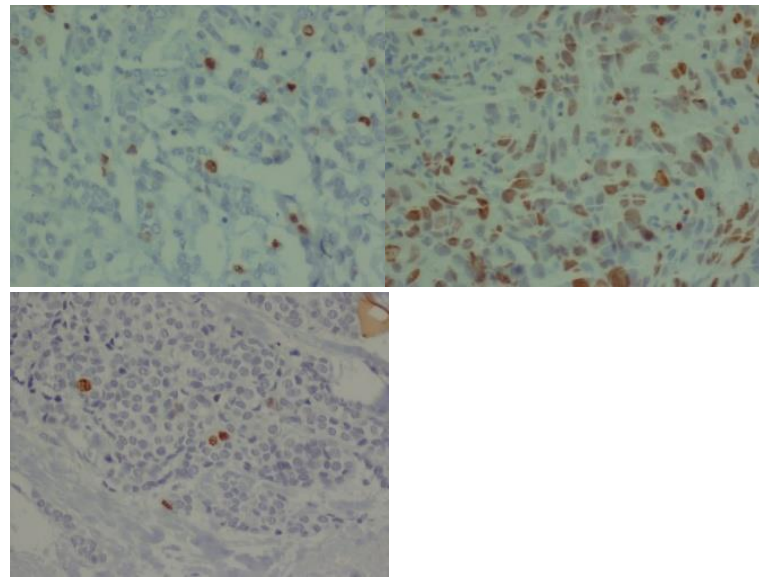

Figure 1. a) A case of IDC with intermediate Ki 67:15-20\% be for neoadjuvant chemotherapy.

b) High Ki $67>30 \%$ be for neoadjuvant chemotherapy c) Low Ki $67<15 \%$ after neoadjuvant chemotherapy

ER and PR were reported as positive or negative status. Her2/neu marker was reported positive when the 3 plus was used for staining, and negative when it was not stained at all or stained with 1plus. If Her2/neu was stained with 2plus, further confirmation was done using molecular test (CISH method). Ki67 marker was reported by counting the number of stained cells and this number was coordinated to all neoplastic epithelial cells. The patients were divided by using subjective light microscope analysis into three groups with low (lower than 15\%), intermediate (16\% to 30\%) and high Ki67 expression (more than 30\%) (18) which is shown in Figures 1-3.

Demographic data, tumor type, the stages and the number of involved lymph nodes were extracted from the patients file. Data were analyzed by SPSS software version 22.0 with independent-sample-T, ANOVA and Tukey tests. 
$\mathrm{P}$-values less than 0.05 were considered as a statistically significant.

\section{Results}

The most common age group were between 41 and 49 years old (32.1\%), followed by $30-39$ years old. The most tumor size was $2-5 \mathrm{~cm}$ (45.5\%) in groups. Also $29.1 \%$ of patients had tumor $2 \mathrm{~cm}$ or less in size and $25.5 \%$ of patients had the tumor larger than $5 \mathrm{~cm}$. Patients were mainly in stage IIIA (40\%) and the following stages were IIB (29.1\%), IIA (14.5\%), IIIC
(9.1\%), I (5.5\%) and finally IIIC (1.8\%). Lymph nodes were involved in most patients (76.4\%). In addition, $60 \%$ of patients were ER positive, $52.7 \%$ were PR positive and nearly half of patients were HER2/neu positive. Most patients (92.7\%) had ductal carcinoma and the next more frequent tumor types were lobular carcinoma (7.3\%).

Among 55 patients, only 29 patients (52\%) had complete response to Neoadjuvant chemotherapy and didn't have any residual tumor after the mastectomy. The association with mean Ki67 level are shown in chart1.

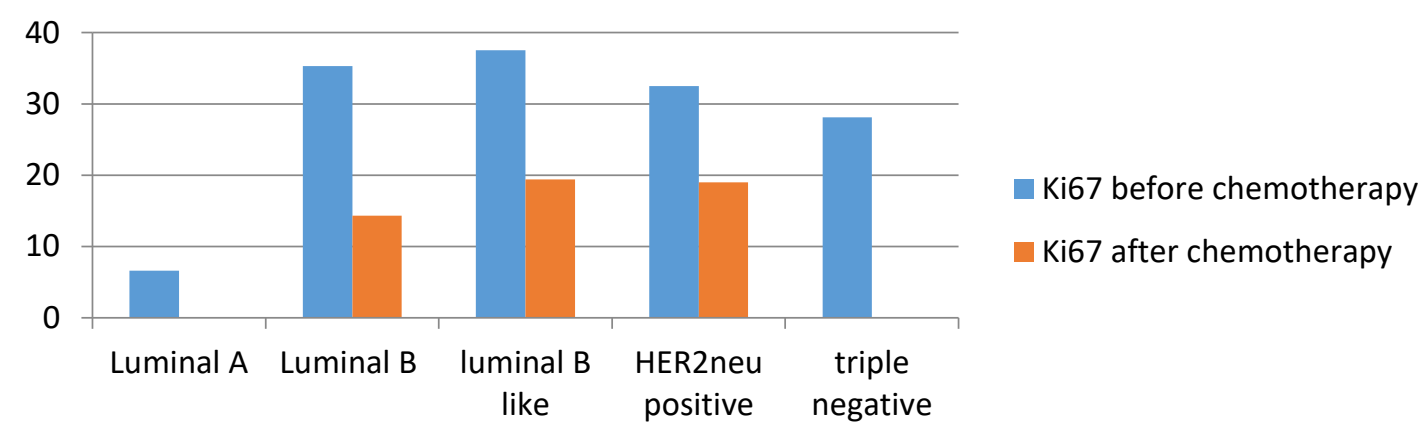

Chart 1. Mean Ki67 level before and after chemotherapy in various subtypes of breast cancer response. Those with a partial response to

The majority of patients $(54.5 \%)$ had high Ki67 expression, $23.6 \%$ of patients had intermediate amount of Ki67 expression and $21.8 \%$ of patients had low Ki67 expression. After treatment, among $52.7 \%$ of patients with complete chemotherapy showed that the most patients (23.6\%) had low Ki67 expression, 20\% had intermediate Ki67 level and 3.6\% had high level of Ki67 that are indicated in chart2.

Table1 Analysis showed that Neoadjuvant chemotherapy causes significant decline in Ki67 expression

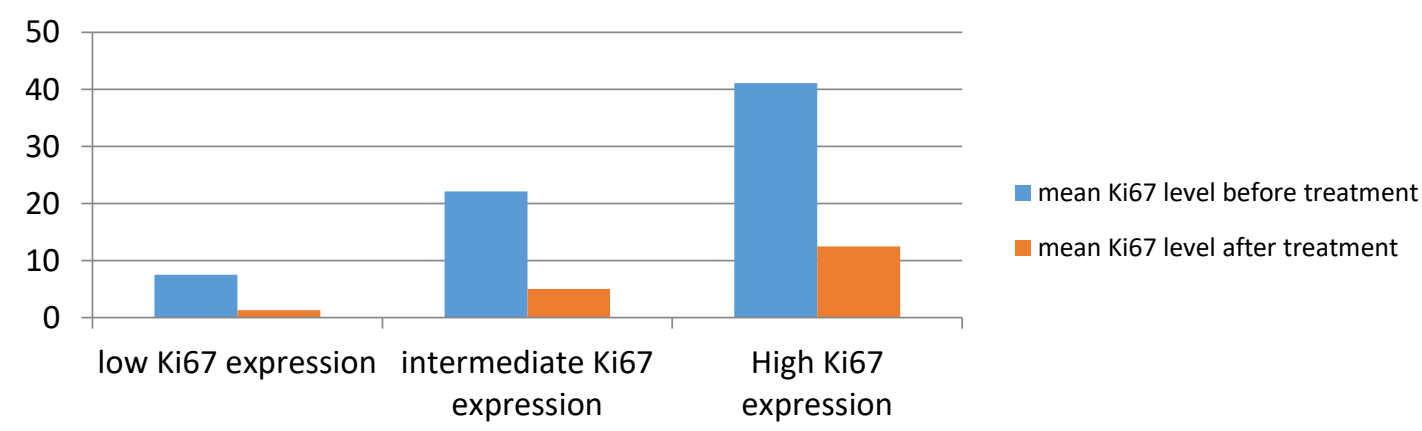

Chart 2. Mean Ki67 level changes before and after treatment in patients with partial response to chemotherapy 
Table 1. Mean Ki67level before and after treatment in Ki67 subgroups

\begin{tabular}{|ccc|}
\hline Ki67 classification & $\begin{array}{c}\text { mean Ki67 before } \\
\text { treatment(percent) }\end{array}$ & $\begin{array}{c}\text { mean Ki67 after } \\
\text { treatment(percent) }\end{array}$ \\
\hline Low Ki67 expression $(<15 \%)$ & 7.5 & $\mathbf{1 . 3}$ \\
\hline Intermediate Ki67 expression $(15 \%<<=29 \%)$ & 22.11 & $\mathbf{5}$ \\
\hline high Ki67 expression $(30 \%=<)$ & 41.1 & $\mathbf{1 2 . 5}$ \\
\hline
\end{tabular}

There was a significant relationship between decline in the amount of Ki67 after chemotherapy and Ki67 level before chemotherapy ( $\mathrm{P}=0.003)$. There was a significant correlation between reductions in the level of Ki67 after treatment and
ER negative and PR status before chemotherapy $(\mathrm{P}=0.01$ and 0.01 respectively). The distribution of mean Ki67 level before and after treatment in various stages of breast cancer are displayed in chart 3.

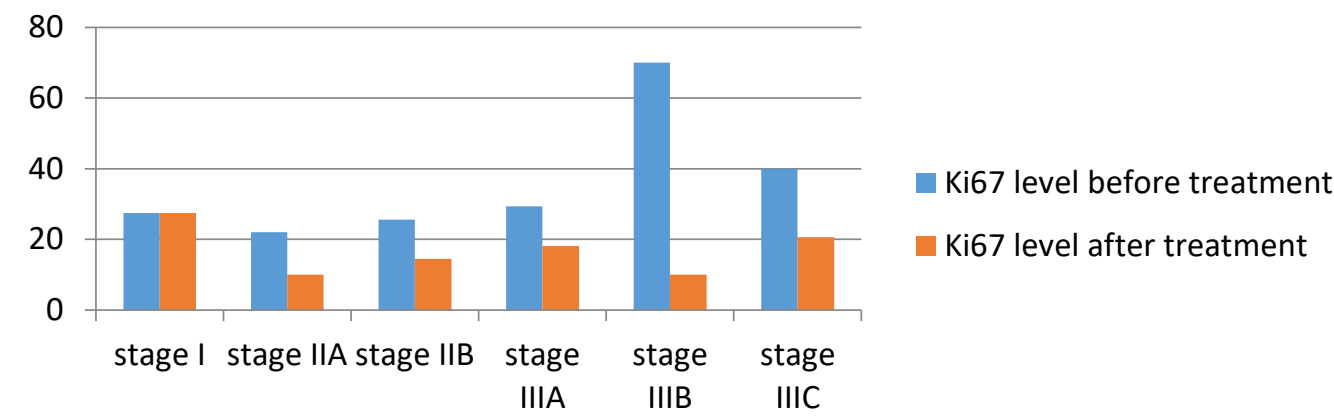

Chart 3. The distribution of mean Ki67 level before and after treatment in various stages of breast cancer

There was no significant correlation between Ki67 level before and after treatment with the tumor size and type. ER and PR positive status were more frequent while Ki67 level before and after treatment was higher in group of metastatic lymph nodes than the ones without involved lymph nodes. HER2/neu expression was higher in group without involved lymph nodes in comparison to metastatic group, but none of the above markers showed significant correlation with metastatic or intact lymph node status. Mean Ki67 level after treatment was significantly lower in triple negative group (0) comparing to other groups (0.87) $(\mathrm{P}=0.0001)$ (chart4).

Ki67 level after the treatment was significantly lower than this level before treatment in this group (P value <0.01) (chart5).

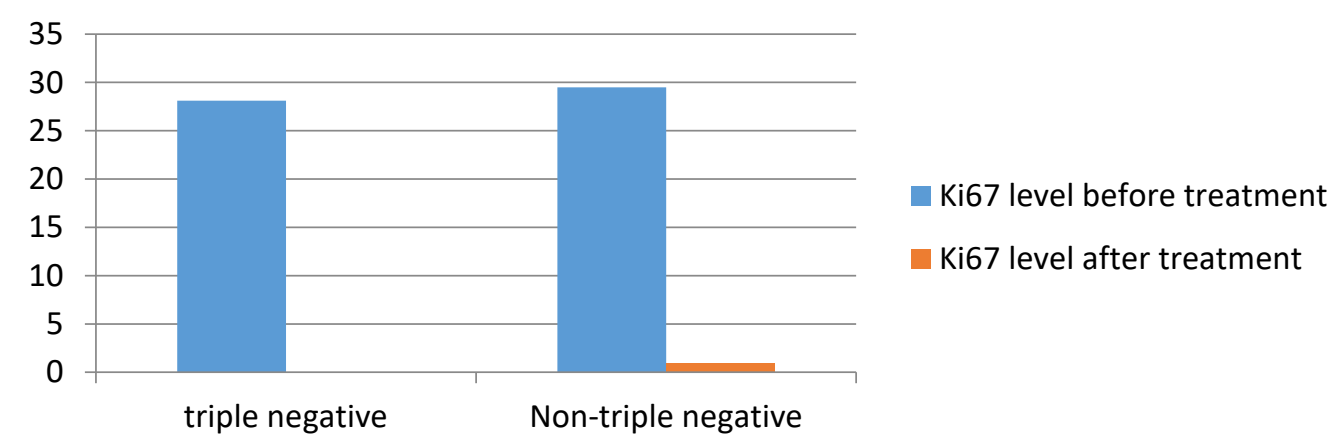

Chart 4. Ki67 before and after treatment in triple negative and Non-triple negative patients 


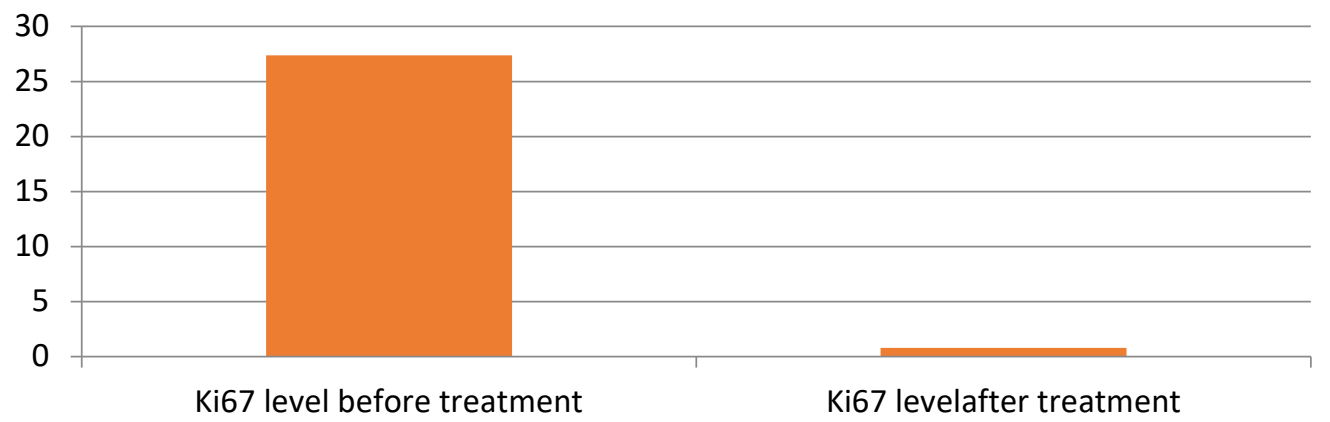

Chart 5. Ki67 before and after treatment in HER2/neu positive patients

\section{Discussion}

Fifty-five female patients enrolled in this study (who were diagnosed by core needle biopsy of breast mass), and then underwent Neoadjuvant chemotherapy before surgery. Most frequent size of tumor was between 2 and $5 \mathrm{~cm}$ (45.5\%) which is in line with the results of Haroon (2013)(16) and Sun (2014) (17). Most participants had ductal carcinoma (90\%) and patients had stage IIIA disease (40\%) and metastatic lymph nodes (76.4\%) which are also consistent with Haroon et al. (17).

Following that, $60 \%$ of the patients were ER positive and $51 \%$ were $\mathrm{PR}$ positive that are completely similar to the study of Mankowitz et al. (14). Most patients (54.5\%) had high Ki67 level, however in all groups with low, intermediate and high Ki67 expression, the Ki67 level was lower after treatment. The analysis indicated that chemotherapy causes significant reduction in Ki67 level of expression which is consistent with the results of Avci's studies (11). In other words, chemotherapy drugs have significant antiproliferative effect on cancerous cells. Thecell destruction as a result of the effect of Anthracylcine on DNA can decrease the newly divided cancer cells which leads to a decline in number of tumor clones (18-25).

After chemotherapy, the reduction of Ki67 level was significantly correlated with ER and PR negative status before chemotherapy ( $\mathrm{P}$ value 0.01 and 0.01 respectively). This correlation was confirmed in Sun and Haroon in 2014 and 2013 $(16,17)$.Also, Sun and his team proved the relationship of Ki67 level after chemotherapy and ER. The significant relationship between Ki67 level before treatment and cancer stage was also observed, which is in line with the results of Inwald's research (26). In both studies, Ki67 levels were lower in lower stages of breast cancer (Sun et al 2013 and Inwald 2013) (16, 26).

In the present study, no relationship was demonstrated between Ki67 level and the number, metastatic and intact status of involved lymph nodes which is contrary to the results of Sun (16) and Haroon (17).

No significant correlation was showed between Ki67 level and tumor size which is in line with the reports of Bonta (24) and Haroon (17), while in 2014, Sun and his colleagues reported this relationship only in early stages of cancer (16). In the group of metastatic lymph nodes, ER and PR positive status were low, whereas Ki67 level was higher than other groups before and after therapy. But Her2/neu indicated the high level of expression in group with intact lymph nodes comparing to the group of metastatic. All the above relationships were not statistically significant. In the present study, 35\% of patients were HER2/neu positive, while in the study of Mirmalek on breast cancer in Iranian population, the reported number for HER2/neu positive status was $58.5 \%$ (27).

In the current study, $52.7 \%$ of patients had complete response, this rate was reported $18 \%$ by Bonnefoi who worked on 1212 cancer patients (28). Triple negative breast cancer is a subtype of breast cancer with poor prognosis because of a high-invasive biological manner and also the absence of endocrine receptors which are the targets of chemotherapy drugs (Tamoxifen and Trastuzumab), But this group responded to chemotherapy better than other subgroups of 
breast cancer (25). In this research study, $14 \%$ of the patients were triple negative which is similar to Boyle's study who showed $14-16 \%$ of triple negative patients (26).

There was not a significant difference between mean Ki67 level before treatment in triple negative group (28.1\%) and other groups (29.5\%) which is in contrast with the results of

Hitao Li's study in 2015. In the present study, Ki67level in triple negative patients $(0 \%)$ was significantly different from the ones in other groups $(0.87 \%)$ after treatment. It can be because all patients in the triple negative group responded to chemotherapy completely while Keam found the same results by analyzing $13.3 \%$ of patients (25). Therefore, our findings confirm the results of the previous studies.

\section{Conclusion}

The results of the present study prove the prognostic significance of decreased ki67 index after Neoadjuvant chemotherapy in breast cancer patients. The level of Ki67 can provide complementary information beside the molecular subtypes and other markers helping to predict the amount of responses to chemotherapy. Based on the findings of this study, Neoadjuvant chemotherapy before mastectomy can help achieve better outcome in cancer patients.

\section{References}

1. Amani D, Hassan ZM, Ravangard F, Frazmand S, Karim-Zadeh M. Flowcytometric Analysis of Tumor Associated Macrophages in Invasive Ductal Carcinoma of Breast. Iran J Immunol. 2005; 2:117-22.

2. Cooksley S, Hipkiss JB, Tickle SP, Holmes-Ievers E, Docherty AJ, Murphy G, et al. Immunoassays for the Detection of Human Collagenase, Stromelysin, Tissue Inhibitor of Metallo-proteinases (TIMP) and EnzymeInhibitor Complexes. Matrix. 1990;10(5):28591.

3. Newman PJ. The Role of PECAM-1 in Vascular Cell Biologya. Annals of the New York Academy of Sciences. 1994; 714(1):16574.

4. Luukkaa $\mathrm{M}$, Vihinen $\mathrm{P}$, Kronqvist $\mathrm{P}$, Vahlberg T, Pyrhönen S, Kähäri VM, et al.
Association between high collagenase-3 expression levels and poor prognosis in patients with head and neck cancer. Head \& neck. 2006;28(3):225-34.

5. Jones RL, Smith IE. Neoadjuvant treatment for early-stage breast cancer: opportunities to assess tumour response. The lancet oncology. 2006;7(10):869-74

6. Kim KI, Lee KH, Kim TR, Chun YS, Lee TH, Park HK. Ki-67 as a predictor of response to neoadjuvant chemotherapy in breast cancer patients. Journal of breast cancer. 2014 Mar 1;17 (1):40-6.

7. Juhasz-Böss I, Mavrova R, Moga S, Radosa J, Schmidt G, Bohle RM, et al. Solomayer E, Herr D. Can Ki-67 Play a Role in Prediction of Breast Cancer Patients' Response to Neoadjuvant Chemotherapy. BioMed Research International. 2014; 2014.

8. Urruticoechea A, Smith IE, Dowsett M. Proliferation marker Ki-67 in early breast cancer. Journal of clinical oncology. 2005; 23(28):7212-20.

9. Archer CD, Parton M, Smith IE, Ellis PA, Salter J, Ashley S,et.al. Early changes in apoptosis and proliferation following primary chemotherapy for breast cancer. British journal of cancer. 2003; 89(6):1035-41.

10. Ellis PA, Smith IE, Detre S, Burton SA, Salter J, A'hern R, et al. Reduced apoptosis and proliferation and increased $\mathrm{Bcl}-2$ in residual breast cancer following preoperative chemotherapy. Breast cancer research and treatment. 1998; 48(2):107-16.

11. Avci N, Deligonul A, Tolunay S, Cubukcu E, Olmez OF, Ulas A, et al. Neoadjuvant chemotherapy-induced changes in immunohistochemical expression of estrogen receptor, progesterone receptor, HER2, and $\mathrm{Ki}$ 67 in patients with breast cancer. J Buon. 2015; 20:45-9.

12. Tanei T, Shimomura A, Shimazu K, Nakayama T, Kim SJ, Iwamoto $\mathrm{T}$, et al. Prognostic significance of Ki67 index after neoadjuvant chemotherapy in breast cancer. Eurp J Surg Oncol. 2011; 37(2):155-61.

13. Jones RL, Salter J, A'Hern R, Nerurkar A, Parton M, Reis-Filho et al. The prognostic significance of Ki67 before and after neoadjuvant chemotherapy in breast cancer. 
Breast cancer research and treatment. 2009; 116(1):53-68.

14. von Minckwitz G, Schmitt WD, Loibl S, Müller BM, Blohmer JU, Sinn BV, et al. Ki67 measured after neoadjuvant chemotherapy for primary breast cancer. Clin Cancer Res. 2013; 19(16):4521-31.

15. Dowsett M, Smith IE, Ebbs SR, Dixon JM, Skene A, A'Hern R, et al. Prognostic value of Ki67 expression after short-term presurgical endocrine therapy for primary breast cancer. Journal of the National Cancer Institute. 2007; 99(2):167-70.

16. Sun J, Chen C, Wei W, Zheng $\mathrm{H}$, Yuan J, Tu Y, et al. Associations and indications of Ki67 expression with clinicopathological parameters and molecular subtypes in invasive breast cancer: A population-based study. Oncol lett. $2015 ; 10(3): 1741-8$.

17. Haroon S, Hashmi AA, Khurshid A, Kanpurwala MA, Mujuba S, Malik B. Ki67 index in breast cancer: correlation with other prognostic markers and potential in pakistani patients. Asian Pac J Cancer Prev. 2013; 14(7):4353-8.

18. Sharifi SN, Sadeghian F, Homaei SF, Haghighi F. Immunohistochemical study of cell proliferation marker (ki-67), estrogen, and progesterone receptors expression in breast carcinoma.Birjand Univ Med Sci. 2006; 13(3): $9-15$.

19. Inic Z, Zegarac M, Inic M, Markovic I, Kozomara Z, Djurisic I, et al. Difference between Luminal A and Luminal B Subtypes According to Ki-67, Tumor Size, and Progesterone Receptor Negativity Providing Prognostic Information. Clin Med Insights Oncol. 2013; 8:107-11.

20. Cheang MC, Chia SK, Voduc D, Gao D, Leung S, Snider J, et al. Ki67 index, HER2 status, and prognosis of patients with luminal $\mathrm{B}$ breast cancer. Journal of the National Cancer Institute. 2009; 101(10):736-50.

21. Keam B, Im SA, Lee KH, Han SW, Oh DY, Kim JH, et al. Ki-67 can be used for further classification of triple negative breast cancer into two subtypes with different response and prognosis. Breast Cancer Res. 2011; 13(2):1-7.

22. Feeley LP, Mulligan AM, Pinnaduwage D, Bull SB, Andrulis IL. Distinguishing luminal breast cancer subtypes by Ki67, progesterone receptor or TP53 status provides prognostic information. Mod Pathol. 2014; 27(4):554-61.

23. Inwald EC, Klinkhammer-Schalke M, Hofstädter F, Zeman F, Koller M, et al. Ki67 is a prognostic parameter in breast cancer patients: results of a large population-based cohort of a cancer registry. Breast Cancer Res. 2013; 139(2):539-52.

24. Bonta I, Bonta D, Loch MM, Eapen A, Blanchard RA. Relationship of Ki67 to tumor size and lymph node metastasis in breast cancer. In ASCO Annual Meeting Proceedings 2012. Vol. 30, No. 15_suppl, p. e21076.

25. Keam B, Im SA, Lee KH, Han SW, Oh DY, Kim JH, et al. Ki-67 can be used for further classification of triple negative breast cancer into two subtypes with different response and prognosis. Breast Cancer Res. 2011; 13(2):1-7.

26. Boyle P. Triple-negative breast cancer: epidemiological considerations and recommendations. Ann Oncol. 2012; 23(suppl 6):vi7-12.

27. Mirmalek SA, Hajilou M, Salimi Tabatabaee SA, Parsa Y, Yadollah-Damavandi $\mathrm{S}$, et al. Prevalence of HER-2 and Hormone Receptors and P53 Mutations in the Pathologic Specimens of Breast Cancer Patients. Int $\mathbf{J}$ Cancer. 2014; 2014.

28. Bonnefoi $\mathrm{H}$, Litière $\mathrm{S}$, Piccart $\mathrm{M}$, MacGrogan G, Fumoleau P, Brain E, et al. Pathological complete response after neoadjuvant chemotherapy is an independent predictive factor irrespective of simplified breast cancer intrinsic subtypes: a landmark and twostep approach analyses from the EORTC 10994/BIG 1-00 phase III trial. Ann Oncol. 2014; 25(6):1128-36.

Moazed V, Jafari E, Kalantari khandani B, Nemati A, benrazavi S. Prognostic Significance of Reduction in Ki67 Index After Neoadjuvant Chemotherapy in Patients With Breast Cancer in Kerman Between 2009 And 2014. Iran J Pathol. 2018; 13(1): 71-78. 